\title{
Interior and H-[?] feedback stabilization for sabra Shell model of turbulence
}

\author{
Tania Biswas ${ }^{1}$ and Sheetal Dharmatti ${ }^{2}$ \\ ${ }^{1}$ IIT Delhi \\ ${ }^{2}$ Indian Institute of Science Education Research Thiruvananthapuram
}

January 15, 2021

\begin{abstract}
Shell models of turbulence are representation of turbulence equations in Fourier domain. Various shell models are studied for their mathematical relevance and the numerical simulations which exhibit at most resemblance with turbulent flows. One of the mathematically well studied shell model of turbulence is called sabra shell model. This work concerns with two important issues related to shell model namely feedback stabilization and robust stabilization. We first address stabilization problem related to sabra shell model of turbulence and prove that the system can be stabilized via finite dimensional controller. Thus only finitely many modes of the shell model would suffice to stabilize the system. Later we study robust stabilization in the presence of the unknown disturbance and corresponding control problem by solving an infinite time horizon max-min control problem. We first prove the $\$ \mathrm{H}^{\wedge} \backslash$ infty $\$$ stabilization of the associated linearized system and characterize the optimal control in terms of a feedback operator by solving an algebraic riccati equation. Using the same riccati operator we establish asymptotic stability of the nonlinear system.
\end{abstract}

\section{Hosted file}

H-infinite stabilization_SD_TB .pdf available at https://authorea.com/users/389701/articles/ 504269-interior-and-h-feedback-stabilization-for-sabra-shell-model-of-turbulence 